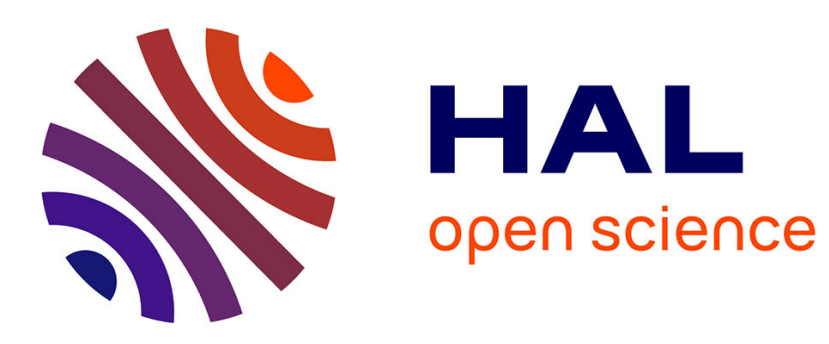

\title{
Convective Flow in a Horizontally Vibrated 3D Granular Packing
}

Ahmed Raihane, Olivier Bonnefoy, Sébastien Nadler, Jean-Louis Gelet, Jean-Marc Chaix, Gérard Thomas

\section{To cite this version:}

Ahmed Raihane, Olivier Bonnefoy, Sébastien Nadler, Jean-Louis Gelet, Jean-Marc Chaix, et al.. Convective Flow in a Horizontally Vibrated 3D Granular Packing. Powders and Grains 2009: 6th International Conference on Micromechanics of Granular Media, Jul 2009, Golden, United States. pp.721-724. hal-00409723

\section{HAL Id: hal-00409723 \\ https://hal.science/hal-00409723}

Submitted on 12 Aug 2009

HAL is a multi-disciplinary open access archive for the deposit and dissemination of scientific research documents, whether they are published or not. The documents may come from teaching and research institutions in France or abroad, or from public or private research centers.
L'archive ouverte pluridisciplinaire HAL, est destinée au dépôt et à la diffusion de documents scientifiques de niveau recherche, publiés ou non, émanant des établissements d'enseignement et de recherche français ou étrangers, des laboratoires publics ou privés. 


\title{
Convective Flow in a Honizontally Vibrated 3D Granular Packing
}

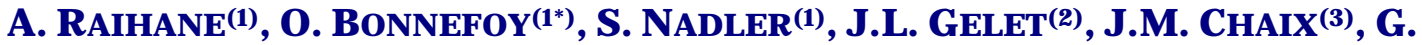 THOMAS(1)}

(1) Ecole nationale supérieure des Mines, Centre SPIN, LPMG-UMR 5148, 158, Cours Fauriel, 42023 Saint-Étienne CX2, France

(2) Ferraz-Shawmut, 6, rue de Vaucanson, 69720 Saint-Bonnet de Mure, France

(3 Centre SIMAP, Grenoble INP-CNRS, 1130 rue de la Piscine, BP 75, 38402 Saint-Martin d'Hères, France

(*) (obonnefoy@emse.fr)

\begin{abstract}
We report experimental observations of a horizontally vibrated granular medium made out of sand grains. For large enough acceleration, two counter-rotating convection rolls appear in the upper part of the granular packing, whereas the bed remains unperturbed in the lower part. For increasing acceleration, the free surface exhibits different shapes: slight dome, two ridges and single roof. A quantitative characterization is performed by mean of PIV and image analysis. It shows that both relaxed and dynamic densities present a maximum at $\Gamma=3.8$ and that the measured thickness of the fluidized region scales with a square root law (as a function of the dimensionless acceleration). In the discussion, a convection mechanism is proposed, as well as an analogy with classical Newtonian fluids.
\end{abstract}

Keywords:

Granular medium ; Powders ; Horizontal Vibration ; Compaction/Densification ; Fluidization ; Grains flow ; Convection rolls pattern, Experimental observations, Numerical modeling, DEM simulation.

PACS:

45.70.-n, 45.70.Cc ; 45.70.Mg, 45.70.Qj, 47.57.Gc, 61.43.Gt, 81.05.Rm, 81.20.Ev, 83.80.Fg, 05.65. $+\mathrm{b}$

\section{Introduction}

Vibration- or shock-based processes are commonly used in industry to densify granular packings and the choice of the process parameters, such as the frequency, RMS or peak acceleration, duration, direction, may appear to be of crucial importance in the final product quality. So it is in the manufacturing process of high intensity electrical fuses developed bythe Ferraz-Shawmut company. These devices are made of a ceramic box containing a silver fuse and filled up with sand grains. The electrical properties of the fuses strongly depend on the granular packing that needs to be as dense and homogeneous as possible.

From an academic point of view, the literature is abundant in works devoted to the influence of taps or vibrations on granular matter. Some important findings are related to vertically excited systems: granular matter may behave as a glass when submitted to periodic shocks, some convective patterns [1] and surface waves [2] may appear in vigorously vibrated cylinders, ratchet effects may be observed in complex geometries [3], segregation through brazil nut effect and its reverse are observed when size and/ or density differences are present [4], etc... This phenomenology is further enriched when the vibrations are horizontal, i.e. orthogonal to the gravity field. In this case, it has been shown that the granular packing may segregate into a lower solid-like zone and an upper fluid-like zone [5].

This study reports experimental observations on a granular medium submitted to horizontal sinusoidal vibrations. It also presents some tentative explanations for the convection mechanism and an analogy with classical fluid (i.e. cohesive). 


\section{EXPERIMENTAL OBSERVATIONS}

In this part, we describe the experimental setup, qualitative features of the grains motion in the bulk and at the surface, data acquisition and processing methods, and finally, quantitative data concerning the evolution of the global density and the velocity field when the acceleration is increased.

\section{Experimental Setup}

As depicted in Fig. 1, the experimental setup is composed of a container fixed on a horizontal mobile table that is driven by an electromagnetic shaker.

The container is made out of Plexiglas ${ }^{\mathrm{TM}}$ so grains are visible through the side walls and their movements can be followed by an ultra-fast camera. The vibrator (Tira TV51110) is delivering a sinusoidal signal characterized by a frequency $\mathrm{f}$ and a dimensionless peak acceleration $\Gamma$ :

$$
x(t)=A \cdot \sin (\omega t) \text { with }\left\{\begin{array}{l}
\omega=2 \pi f \\
\Gamma g=A \omega^{2}
\end{array}\right.
$$

A lot of care has been brought to reduce to negligible values the friction between the sliding table and the marble stand and the misalignment between the table and the shaker vibration axis. The granular matter is made out of rounded sand grains (silica), which Particle Size Distribution is polydisperse enough to prevent any crystallization. The main data are in Table 1.

\section{Sample Preparation}

Two protocols have been used to prepare granular beds with a homogeneous structure and a specified packing fraction.

The first protocol (\#1) leads to a density of approximately $61.5 \%$. It consists in pouring sand grains into the container directly from a funnel (min. diameter of $8 \mathrm{~mm}$ ) situated $\sim 50 \mathrm{~mm}$ above the free surface. During the filling, the funnel is moved here and there to avoid the formation of a cone.

The second protocol (\#2) leads to a density of approximately 68\%. It consists in pouring the sand grains over a mesh (aperture of $2 \mathrm{~mm}$ ) situated $\sim 100 \mathrm{~mm}$ above the free surface. This way, grains fall with a controlled velocity and are uniformly spread over the container surface (uniform rain) rather than flowing out of a localized source.

In the reported experiments, the two protocols are used. The default initial bed height is 60 $\mathrm{mm}$ and the default frequency is $50 \mathrm{~Hz}$.

\section{Phenomenology}

Visual observations of grain motions indicate different behaviors when the initially immobile container is vibrated with an increasing acceleration.

For $0<\Gamma<0.4$, the structure of the granular packing remains the same as in the initial state, as if it was a solid. For $0.4<\Gamma<1.35$, some simmering is observed in the two or three upper grain layers.

For $1.35<\Gamma<6.5$, the bed is divided into two distinct zones. In the lower region, the granular medium still behaves like a solid. In the upper region, it is fluidized and we observe two stationary convection rolls (see Fig. 2 and 3). With increasing acceleration, the convection becomes stronger and the fluidized region expands at the detriment of the solid-like region. The initially flat free surface turns into a slight dome, then two ridges that progressively merge into a single roof (see Fig. 4).

For $\Gamma>6.5$, the invariance of the convective pattern by translation along the transverse y-axis is lost and we can observe strong downwards fluxes along the four vertical corners. 


\section{Quantitative characterization}

\section{Data Acquisition and Processing}

For each experiment $(\Gamma, \mathrm{f})$, the global density was measured before, during, and after vibrations, and the ultra-fast camera recorded the positions of the grains situated along the transparent North face. A PIV software was specially developed, the image analysis being based on a differential method assuming the optical flow conservation. For each pair of consecutive images, the displacement of the grains is calculated and the multi-scale, coarseto-fine, analytical approach gives access to robust data even in the presence of strong deformations. We have validated this method in different configurations: pure translation and pure rotation for numerical deformation or physical deformation (noise due to lighting fluctuations). For each experiment, the velocity field is averaged over 10 pairs of images, and, when correctly calibrated, the maximum error is estimated at $5 \%$ [6].

\section{Influence of the Acceleration on the Global Density}

As shown in Fig. 5, the horizontal vibration of a sample prepared with protocol \#1 leads to a strong densification. It can be seen that the densification rate is maximum for $\Gamma \sim 3.8$. The granular packing gets further densified when the vibrations stop. Other experimental results (not reported here because of lack of space) give access to the local density profiles. They reveal that the densification process occurs rather homogeneously in the whole bed. In the solid-like region, the densification by grain rearrangement happens in some hundreds of periods.

\section{Influence of the Acceleration on the Velocity Field}

Since the velocity field on the North face is invariant by translation along the transverse yaxis, we post-processed it in order to compute the vertical component of the grain velocity at any altitude of the median (xz) plan (e.g. along the vertical thick black line represented in Fig. 3). Some of these velocity profiles are reported in Fig. 6.

These profiles confirm the existence of the two regions observed visually: the lower region, with vanishingly small velocity, is the signature for a solid-like granular medium, whereas the upper region, with velocities reaching up to $\sim 47 \mathrm{~mm} \mathrm{~s}^{-1}$ for $\Gamma=6.3$, is the signature of a fluidized region with convective flow. If we arbitrarily define the solid/ fluid transition with a velocity criterion, it becomes possible to calculate the thickness of the fluidized region as a function of the acceleration. A sensibility analysis has shown that the three thresholds 0.02, 0.06 and $0.18 \mathrm{~mm} \mathrm{~s}^{-1}$ give results in agreement with the visual observations and that the calculated thickness T varies by no more than $2 \mathrm{~mm}$, when the threshold is changed. In the following, we adopt a threshold value of $0.06 \mathrm{~mm} \mathrm{~s}^{-1}$. On Fig.7, we report experimental values obtained for different accelerations and initial bed heights.

It appears, that for $\Gamma>\Gamma_{\mathrm{c}}$ all data collapse onto a master curve, which equation may be fitted by a square root law until the fluidized thickness saturates at a level fixed by the initial bed height Hi:

$$
T(\Gamma)=T^{*} \cdot \sqrt{\Gamma-\Gamma_{c}} \text { with }\left\{\begin{array}{l}
T^{*}=17 \mathrm{~mm} \\
\Gamma_{c}=1.35
\end{array}\right.
$$

Other experimental measurements made on samples prepared with protocols \#1 and \#2 show that the fluidized thickness does not depend neither on the initial density (in the range 61$67 \%$ ), nor on the frequency (in the range $50-85 \mathrm{~Hz}$ ). At first sight, these data are similar to the data obtained by Metcalfe et al.[5]. However, it is difficult to draw clear conclusions because

1- frequency and acceleration ranges are quite different

2- the fluidized thickness is measured on the North Face in our work and on the East Face in their work 
3- grains rise along the East-West axis in our experiments (axial symmetry) and in the middle of the cell in their experiments (central symmetry).

\section{Discussion and Fluid Analogy}

Experiments reported above show that a granular medium submitted to horizontal sinusoidal vibrations may segregate into a lower solid-like region and an upper fluidized region, where two stationary counter-rotating rolls are observed. Observations suggest that the mechanism for this convection pattern may be the following: because of inertia, a temporary "gap" is created between the bulk of the granular medium and the North and South face alternatively. Grains fall into the gap by gravity, until they reach its bottom. On the other hand, the vibrations induce an average horizontal compressive stress that turns into shear and, consequently, into an ascendant grains movement in the central part of the packing. Preliminary works have shown that a fluid analogy may be of interest. Indeed, apart from the gravity field, only one horizontal constant force $\mathrm{F}$ is necessary to generate convection patterns in a Newtonian liquid. Fig. 8 shows two convection rolls obtained with a volume force F, where $a$ and $b$ are positive constants:

$$
\left\|\vec{F}_{(x)}\right\| \propto \exp \left(-a .\left|x-\frac{L_{x}}{2}\right|-b z\right)
$$

In this example, the driving force is non negligible only in the immediate vicinity of the North and South walls and its intensity decreases when approaching the liquid surface. Similarly, one can imagine that, due to mechanical energy dissipation during the shocks, the equivalent pressure exerted by the walls on the grains decreases, when moving away from the walls. This could be also the case when going up closer to the free surface, where attenuated stress transmissions are attributed to lower density. Even if this analogy remains questionable because granular matter does not show cohesive behaviour, it should be seen as a tool to understand the behaviour of a granular medium submitted to horizontal vibrations.

\section{References}

[1] E. Rouèche, "Influence des paramètres de vibrations sur la rhéologie d'un milieu granulaire. Application au remplissage des fussibles", Ph.D. Thesis, Ecole nationale supérieure des Mines de Saint-Etienne, France, 2005.

[2] HK. Pak and R.P. Behringer, Phys. Rev. Letters 71, 12 (1993)

[3] D. Van der Meer et al., Phys. Rev. Letters 92, 18 (2004)

[4] M.E. Mobius et al., Nature 414, 6861(2001)

[5] S.G.K. Tennakoon, Europhysics Letters 45, 470 (2005)

[6] J. Debayle et al., IAS, submitted (2008)

\section{Figures captions}

Figure 1: Sketch of the experimental setup.

Figure 2: East face view of the lower solid-like zone (red-colored and natural sand grains keep immobile) and the upper fluid-like zone (convective motion leads to mixing). Observation made for $1.35<\Gamma<6.5, \mathrm{f}=50 \mathrm{~Hz}$. Sample prepared with protocol \#2.

Figure 3: Two parallel counter-rotating convection rolls observed for $1.35>\Gamma<6.5, \mathrm{f}=50 \mathrm{~Hz}$.

Figure 4: Evolution of the morphology of the free surface (East side view) for increasing acceleration: slight dome $\rightarrow$ slight dome +2 bumps $\rightarrow$ chalet roof.

Figure 5: Initial, dynamic and final global densities as a function of the applied acceleration, $\mathrm{f}=50 \mathrm{~Hz}$. Samples prepared with protocol \#1.

Figure 6: Velocity profile $v z=f(z)$ along the North face for three accelerations, $f=50 \mathrm{~Hz}$. Samples prepared with protocol \#1. 
Figure 7: Fluidized thickness T as a function of the acceleration for different initial sand heights (Hi). Samples prepared with protocol \#1.

Figure 8: Slight dome and two stationary counter-rotating convection rolls observed in a Newtonian liquid (stream lines are manually thickened for purpose of clarity).

\section{Tables captions}

Table 1: Main experimental characteristics.

\section{Figures}

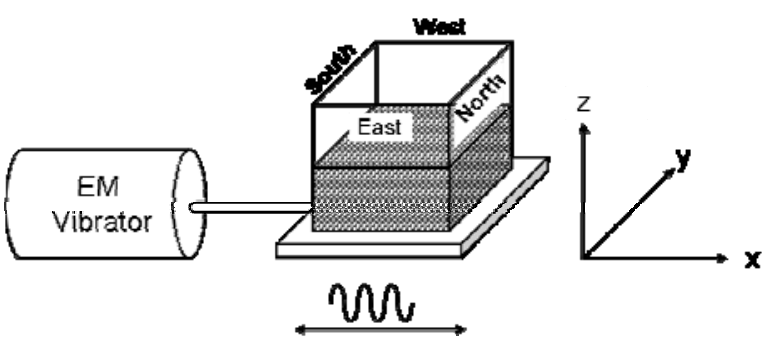

Figure 1

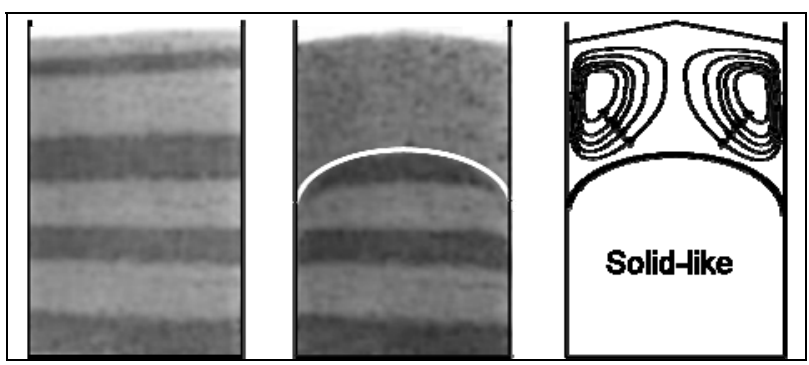

Figure 2

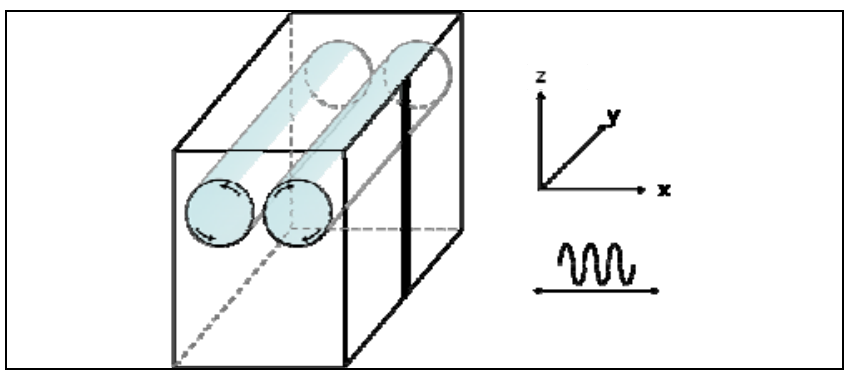

Figure 3

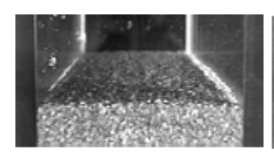

$\Gamma=1.4$

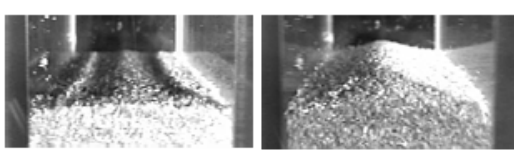

$\Gamma=3.5$
$\Gamma=6.2$

Figure 4 
Powders and Grains 2009 Golden, Colorado : 13 au 17juillet 2009 ISBN 978-0-7354-06827721- 724

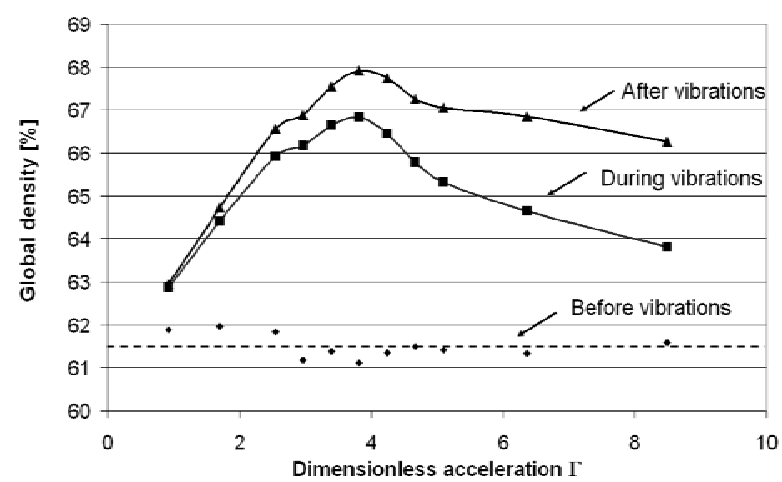

Figure 5

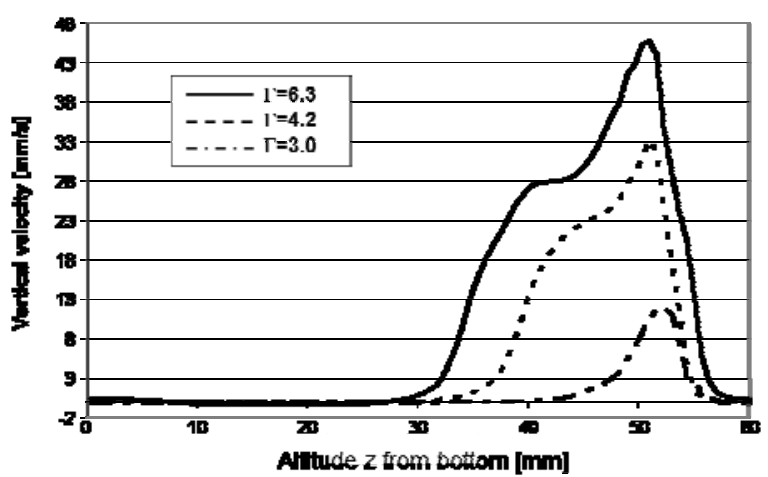

Figure 6

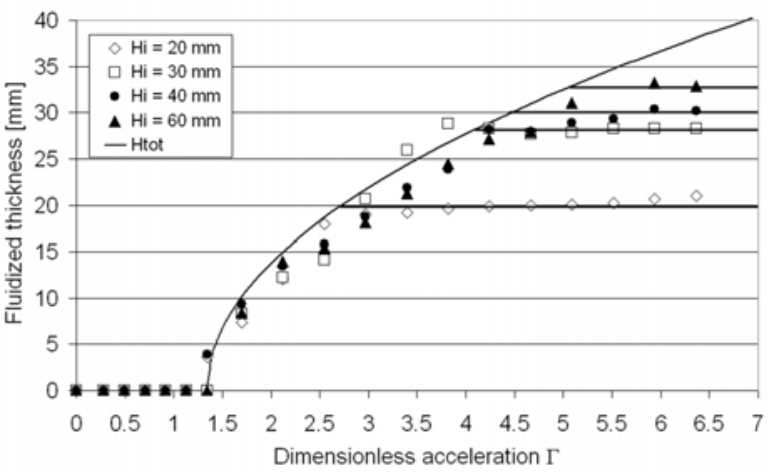

Figure 7

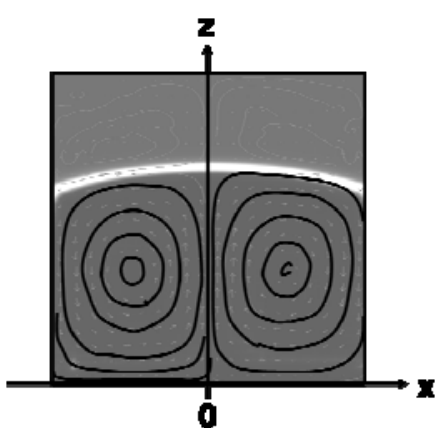

Figure 8 
Powders and Grains 2009 Golden, Colorado : 13 au 17juillet 2009 ISBN 978-0-7354-06827721- 724

\section{Table 1}

\begin{tabular}{|ccc|}
\hline Parameter & Value & Unit \\
\hline Grains Sauter diameter d & 0.5 & $\mathrm{~mm}$ \\
\hline Grains PSD span & 0.68 & - \\
\hline Grains density & 2700 & $\mathrm{~kg} \mathrm{~m}^{-3}$ \\
\hline Container Length Lx & 40 & $\mathrm{~mm}$ \\
\hline Container Width Ly & 80 & $\mathrm{~mm}$ \\
\hline Container Height Lz & 80 & $\mathrm{~mm}$ \\
\hline Initial sand bed thickness & $20-60$ & $\mathrm{~mm}$ \\
\hline Dimensionless acceleration $\Gamma$ & $0-8$ & - \\
\hline Frequency f & $50-85$ & $\mathrm{~Hz}$ \\
\hline
\end{tabular}

